\title{
A FUNCTIONAL GENERALIZATION OF DIAMOND- $\alpha$ INTEGRAL MINKOWSKI'S TYPE INEQUALITY ON TIME SCALES
}

\author{
GuAng-Sheng Chen And Pin WAng
}

Abstract. In this paper, we establish a functional generalization of Minkowski's type inequality on time scales based on diamond- $\alpha$ integral, which is introduced as a linear combination of the delta and nabla integrals. Some related results are also obtained.

Mathematics subject classification (2010): 26E70, 26D15. equality.

Keywords and phrases: Diamond- $\alpha$ integral, time scale, Hölder's inequality, Minkowski's type in-

\section{REFERENCES}

[1] H. Minkows KI, Geometrie der Zahlen, Teubner, Leipzig, 1910.

[2] W. Rudin, Real and Complex Analysis, third ed., McGraw-Hill, New York, 1987.

[3] H. Agahi, R. Mesiar, Y. Ouyang, General Minkowski type inequalities for Sugeno integrals, Fuzzy Sets and Systems 161 (2010), 708-715.

[4] J. Y. LU, K. S. WU, J. C. LIN, Fast full search in motion estimation by hierarchical use of Minkowski's inequality, Pattern Recognition 31 (1998), 945-952.

[5] S. HiLger, Analysis on measure chains - a unified approach to continuous and discrete calculus, Results Math. 18 (1990), 18-56.

[6] R.P. Agarwal, M. Bohner, Basic calculus on time scales and some of its applications, Results Math. 35, 1-2 (1999), 3-22.

[7] F. M. Atici, G. S. Guseinov, On Green's functions and positive solutions for boundary value problems on time scales, J. Comput. Appl. Math. 141 (2002), 75-99.

[8] M. Bohner, A. Peterson, Dynamic Equations on Time Scales: An Introduction with Applications, Birkhäser, Boston, 2001.

[9] M. Bohner, A. Peterson, Advances in Dynamic Equations on Time Scales, Birkhäser, Boston, 2002.

[10] Q. Sheng, M. FAdAg, J. Henderson, J. M. Davis, An exploration of combined dynamic derivatives on time scales and their applications, Nonlinear Anal., Real World Appl. 7 (2006), 395-413.

[11] J. W. Rogers JR., Q. Sheng, Notes on the diamond- $\alpha$ dynamic derivative on time scales, J. Math. Anal. Appl. 326, 1 (2007), 228-241.

[12] A. B. MalinowskA, D. F. M. Torres, On the diamond- $\alpha$ Riemann integral and mean value theorems on time scales, Dyn. Syst. Appl. 18, 3-4 (2009), 469-482.

[13] F. H. Wong, C. C. YeH, S. L. YU, C. H. Hong, Young's inequality and related results on time scales, Appl. Math. Lett. 18 (2005), 983-988.

[14] F. H. Wong, C. C. YeH, W. C. LiAn, An extension of Jensen's inequality on time scales, Adv. Dyn. Syst. Appl. 1, 1 (2006), 113-120.

[15] U. M. ÖZKAN, M. Z. SARIKAYA, H. YILDIRIM, Extensions of certain integral inequalities on time scales, Appl. Math. Lett. 21, 10 (2008), 993-1000.

[16] G. S. Chen, Some improvements of Minkowski's integral inequality on time scales, Journal of Inequalities and Applications 2013 (2013), 318.

[17] Moulay R. S. Ammi, Rui A. C. Ferreira, Delfim F. M. Torres, Diamond- $\alpha$ Jensen's inequality on time scales, J. Inequal. Appl. (2008), doi:10.1155/2008/576876. Article ID 576876, 13 pages. 
[18] W. G. YANG, A functional generalization of diamond- $\alpha$ integral Holder's inequality on time scales, Appl. Math. Lett. 23 (2010), 1208-1212.

[19] R. Bibi, M. Bohner, J. PeČARIĆ, And S. VARošAnec, Minkowski and Beckenbach-Dresher inequalities and functionals on time scales, Journal of Mathematical Inequalities 7, 3 (2013), 299-312. 